\title{
Ileal Stenosis, CTCAE
}

National Cancer Institute

\section{Source}

National Cancer Institute. Ileal Stenosis, CT CAE. NCI Thesaurus. Code C143578.

A disorder characterized by a narrowing of the lumen of the ileum. 